Atmospheric pressure ionization waves propagating through a flexible high aspect ratio capillary channel and impinging upon a target

This article has been downloaded from IOPscience. Please scroll down to see the full text article.

2012 Plasma Sources Sci. Technol. 21034001

(http://iopscience.iop.org/0963-0252/21/3/034001)

View the table of contents for this issue, or go to the journal homepage for more

Download details:

IP Address: 141.211.173.82

The article was downloaded on 26/06/2013 at 15:42

Please note that terms and conditions apply. 


\title{
Atmospheric pressure ionization waves propagating through a flexible high aspect ratio capillary channel and impinging upon a target
}

\author{
Zhongmin Xiong and Mark J Kushner ${ }^{1}$ \\ Electrical Engineering and Computer Science Department, University of Michigan, 1301 Beal Ave, \\ Ann Arbor, MI 48109-2122, USA \\ E-mail: zxiong@umich.edu and mjkush@umich.edu
}

Received 6 September 2011, in final form 8 December 2011

Published 16 April 2012

Online at stacks.iop.org/PSST/21/034001

\begin{abstract}
Atmospheric pressure ionization waves (IWs) propagating in flexible capillary tubes are a unique way of transporting a plasma and its active species to remote sites for applications such as biomedical procedures, particularly in endoscopic procedures. The propagation mechanisms for such IWs in tubes having aspect ratios of hundreds to thousands are not clear. In this paper, results are discussed from a numerical investigation of the fundamental properties of ionization waves generated by nanosecond voltage pulses inside a $15 \mathrm{~cm}$ long, $600 \mu \mathrm{m}$ wide (aspect ratio 250), flexible dielectric channel. The channel, filled with a $\mathrm{Ne} / \mathrm{Xe}=99.9 / 0.1$ gas mixture at $1 \mathrm{~atm}$, empties into a small chamber separated from a target substrate by $1 \mathrm{~cm}$. The IWs propagate through the entire length of the channel while maintaining similar strength and magnitude. Upon exiting the channel into the chamber, the IW induces a second streamer discharge at the channel-chamber junction. This streamer then propagates across the chamber and impinges upon the target. The average speeds of the capillary-bounded IW are about $5 \times 10^{7} \mathrm{~cm} \mathrm{~s}^{-1}$ and $1 \times 10^{8} \mathrm{~cm} \mathrm{~s}^{-1}$ for positive and negative polarities, respectively. The propagation speed is sensitive to the curvature of the channel. In both cases, the peak in ionization tends to be located along the channel walls and alternates from side-to-side depending on the direction of the local instantaneous electric field and curvature of the channel. The ionization region following the IW extends up to several centimeters inside the channel, as opposed to being highly localized at the ionization front in unconstrained, atmospheric pressure IWs. The maximum speed of the IW in the chamber is about twice that in the channel.
\end{abstract}

(Some figures may appear in colour only in the online journal)

\section{Introduction}

Combining therapeutic effects and minimum tissue damage, cold, atmospheric pressure plasmas (CAPPs) are being investigated for many biomedical applications such as sterilization, wound healing, cell manipulation and cancer therapy [1-4]. The generation and propagation of CAPPs in long, flexible capillary tubes having large aspect ratios not only

\footnotetext{
1 Author to whom any correspondence should be addressed.
}

have direct applications, such as catheter sterilization, but are also an important way of transporting a plasma and its active species to remote targeted areas [5-7]. When incorporated with various endoscopic devices, remotely delivered plasmas through capillary tubes potentially open new doors for minimally invasively treating disease inside the human body with CAPPs.

Atmospheric pressure ionization waves (IWs) underlie the operation of many CAPP devices including plasma jets [2-4]. 
Although the discharges in such devices typically appear as a luminous plasma continuum, high-speed imaging shows that the plasma in fact consists of discrete, fast-traveling, plasma bullets [8-10], or IW fronts. The propagation of the IWs is responsible for transporting the associated intense electric field, and creating charged and neutral excited species and UV photons at remote locations. One such manifestation is the propagation of an IW through a capillary tube having diameters of less than a few millimeters and aspect ratios of hundreds to thousands. With properly chosen capillary tubes, the distance can be of tens of centimeters from the location at which the plasma is initiated to the target areas [5,6]. The dynamics of the capillary-bounded CAPP IWs are not well understood, although there are clear similarities with atmospheric pressure streamer discharges. For example, the propagation speeds of the IWs are similar. However, the distance and circuitous path that IWs travel in capillary tubes, with little apparent change from the source to the target, are distinct properties.

Much of the understanding of IWs in bounded systems has come from theoretical and experimental studies in straight, shielded discharge tubes, typically having a diameter of a few centimeters and filled with noble gas at low pressures (1-10 Torr) [11-13]. The speed of the IWs can be as high as $5 \times 10^{9} \mathrm{~cm} \mathrm{~s}^{-1}$, and the peak speed is typically reached at a gas pressure of around 5 Torr [14]. At atmospheric pressure, the width of the ionization front or streamer head is on the order of a few hundred micrometers and the propagation speed is typically between $10^{6}$ and $10^{8} \mathrm{~cm} \mathrm{~s}^{-1}$. Kim et al [5] investigated a He plasma jet exiting from a biocompatible flexible tube of $254 \mu \mathrm{m}$ diameter. The plasma exits the tube as a discrete IW front, often called a plasma bullet. They found the speed of the plasma bullets to be (3-6) $\times 10^{7} \mathrm{~cm} \mathrm{~s}^{-1}$. Robert et al [6] developed a compact nanosecond dielectric barrier discharge (DBD) inside capillaries of diameter $200 \mu \mathrm{m}-4 \mathrm{~mm}$ flushed with neon or helium. They found that the plasma bullets propagate at speeds up to $5 \times 10^{8} \mathrm{~cm} \mathrm{~s}^{-1}$ over distances of a few tens of centimeters and exit from the capillary tube as a streamer discharge. A few centimeters downstream of the DBD source, the IW appears to be electronically isolated from the high-voltage source.

Due to the small dimension and high propagation speed, detailed measurements and characterization of atmospheric pressure discharge streamers, particularly in confined geometries, are experimentally difficult. Numerical simulations have been useful in understanding the dynamics of CAPP IWs. Luque et al [15] investigated the evolution and speeds of positive and negative streamers in ambient air and found that the propagation speed of the negative streamers is lower than their positive counterparts. This was explained by electron diffusion in the negative streamer case which tends to broaden the electric field at the streamer head. Naidis [16,17] also numerically investigated the evolution of the ionization front in helium plasma jets propagating into air and also found polarity-dependent effects. In semienclosed space, Georghiou et al [18] investigated atmospheric pressure streamer propagation in a point-to-plane geometry with the streamer entering into a dielectric tube of radius $200 \mu \mathrm{m}$. They found that the propagation speed increased with increasing dielectric constant of the tube, a consequence of the dielectric being in series with the applied voltage. Jansky et al $[19,20]$ numerically and experimentally investigated atmospheric pressure air discharges initiated by a needle anode inside a capillary tube up to $1 \mathrm{~cm}$ in length. They found that the plasma behind the discharge front can have two modes, homogeneous or tubular, depending on the inner radius of the tube and the dielectric constant of the wall. The experiment also showed that for a tube radius of $100 \mu \mathrm{m}$, the discharge propagation speed was 3-4 times higher than the speed obtained without the tube.

The goal of the study discussed in this paper is to clarify the mechanisms of propagation of IWs inside capillary tubes of arbitrary length and path (e.g. tubing with loops and bends). As a first step toward understanding the properties of these discharges, we discuss results from numerical simulations of CAPP IWs propagating through a long, flexible capillary channel connected to a target chamber, both filled with a $\mathrm{Ne} / \mathrm{Xe}=99.9 / 0.1$ mixture at $1 \mathrm{~atm}$ pressure. The IWs, initiated at one end of the channel by a nanosecond highvoltage pulse, propagate through the entire length of the capillary channel without significant change in speed, strength and magnitude of densities. The IWs exiting the tube induce a streamer discharge in the target chamber which then propagates across the chamber and impinges upon a target surface. In section 2, we first describe the discharge configuration and briefly discuss the numerical simulation method. The features of the IWs, of both positive and negative polarities, propagating inside the capillary tube and the target chamber are presented in section 3. Our concluding remarks are in section 4.

\section{Description of the model and discharge configuration}

The numerical modeling platform used in this study is nonPDPSIM, a two-dimensional (2D) plasma hydrodynamics model with radiation-photon transport. It is the same model as that used for the investigation of surface discharges discussed in [21]. Continuity equations for charged and neutral species, and Poisson's equation are solved coincident with the electron energy equation with transport coefficients obtained from solutions of Boltzmann's equation. The photon transport is based on a propagator or Green's function method which accounts for obstructions. The spatial discretization is based on finite volume methods using an unstructured mesh. The time advancement is implemented with a fully implicit Newton iteration method. The channel and the target chamber are filled with $\mathrm{Ne}$ at $1 \mathrm{~atm}$ with a trace impurity represented by Xe at the level of $0.1 \%$. The photoionization of Xe by the UV light from the dimer $\mathrm{Ne}_{2}^{*}$ provides seeding electrons in front of the ionization front, which for the positive polarity case enables the IW to propagate. Details of the numerical methods and $\mathrm{Ne} / \mathrm{Xe}$ reaction mechanism are in [21].

Clearly, IWs in flexible and circuitous tubes have characteristics that depend on the asymmetries of the threedimensional (3D) geometry. nonPDPSIM, being a 2D simulation, is only able to address channels (and not tubes) which have circuitous paths. Although details of IWs 
propagating in truly $3 \mathrm{D}$ tubes will differ from those in channels, we believe the fundamental characteristics are essentially the same. For example, we have recently compared 2D simulations of IWs with the experiments in a 3D rectangular channel with the same width and less than an aspect ratio of two in the channel. We found quantitative agreement in both the wave speed and the electric field at the ionization front [22].

The powered and grounded electrodes, dielectric capillary channel and the target chamber used in this study are schematically shown in figure 1. This geometry is intended to emulate the experiments of Roberts et al [6]. The capillary channel extends from a DBD plasma source to a target chamber. The channel is $600 \mu \mathrm{m}$ wide and about $15 \mathrm{~cm}$ long (aspect ratio 250). In addition to the straight section connected to the target chamber, the channel consists of seven sections with different radii of curvature. The radii of curvature are $0.125,0.5,1.0,1.5,-1.0,0.5-0.5 \mathrm{~cm}$, from the beginning of the channel at the bottom to the top end. The curvature is defined as positive if the unit tangent vector rotates counterclock wise along the curve. Eight locations A-H are labeled along the channel for convenient reference of the instantaneous IW locations later. The channel wall has a uniform thickness of $600 \mu \mathrm{m}$ and a dielectric constant $\varepsilon_{\mathrm{r}}=4$. The computational domain outside the capillary tube has the dielectric properties of air, $\varepsilon_{\mathrm{r}}=1$.

The plasma is initiated at the beginning of the channel where grounded metal electrodes bound the channel. A metal needle is inserted into the channel as the powered electrode in a DBD configuration. The needle is about $800 \mu \mathrm{m}$ long with a radius of curvature at the tip of $25 \mu \mathrm{m}$. The target chamber is $3 \mathrm{~cm}$ wide and $1 \mathrm{~cm}$ high between the end of the channel and the dielectric target surface. The full computational domain is a $12 \mathrm{~cm}$ square and is grounded on the side $(x=0$ and $12 \mathrm{~cm})$ and bottom $(y=0 \mathrm{~cm})$ boundaries. The top boundary $(y=12 \mathrm{~cm})$ consists of the target layer which is $2 \mathrm{~mm}$ thick and has the same dielectric properties as the channel wall. The unstructured computational grid has a total of 23000 nodes and is locally refined inside the plasma zone (channel and target chamber), where the mesh size varies between 10 and $8 \mu \mathrm{m}$. The initial electron density is zero everywhere except at a small spot of the plasma near the needle tip, $10^{12} \mathrm{~cm}^{-3}$ in density and $300 \mu \mathrm{m}$ in diameter. A constant high-voltage $(25-50 \mathrm{kV})$ pulse of either positive or negative polarity is applied to the needle with a pulse rise time of $20 \mathrm{~ns}$. The total simulation time is $300 \mathrm{~ns}$.

\section{IWs in the capillary channel}

For both positive and negative polarities, IWs, initiated at the start of the channel, are able to propagate through the entire $15 \mathrm{~cm}$ channel length while maintaining nearly constant plasma characteristics and speed within a factor of two. The IW exiting the capillary tube triggers a conventional streamer inside the target chamber which then impinges onto the surface. Upon striking the surface the IW splits into two side-traveling ionization fronts. Electron density contours for the positive IW and streamer discharge before the impingement onto the surface are shown in figure 2. The electron density is shown
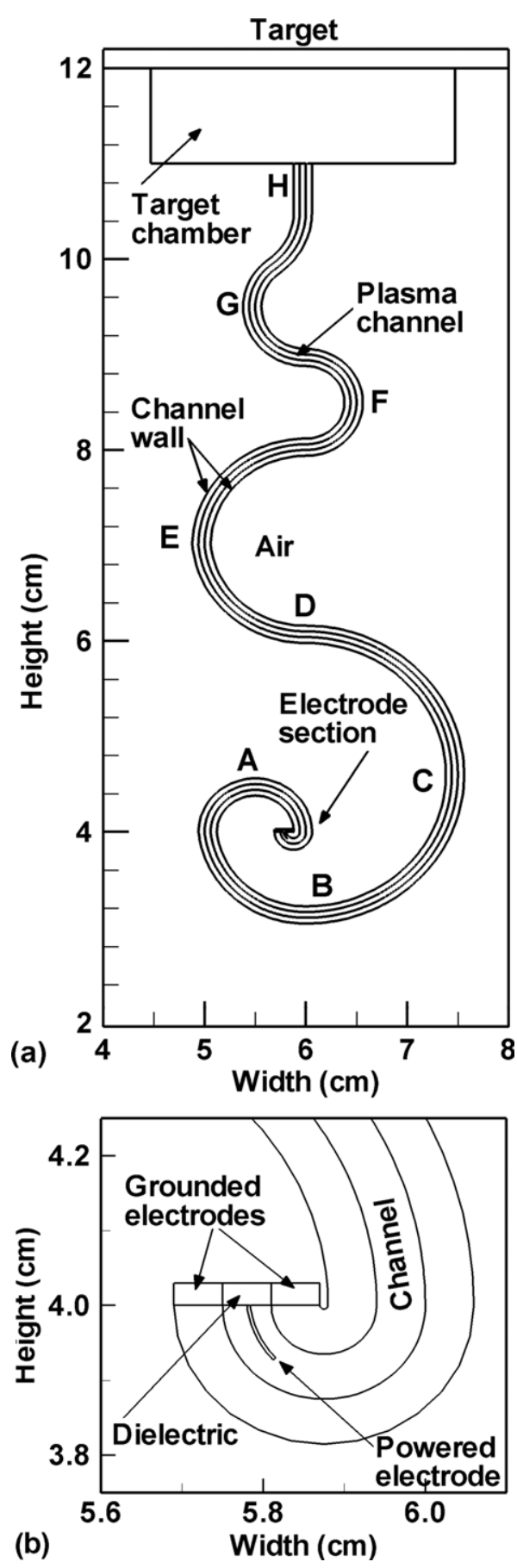

Figure 1. (a) Discharge configuration of ionization waves propagating through a flexible, dielectric capillary channel and impinging upon a target. $(b)$ The ionization waves are initiated by a DBD consisting of a needle powered electrode and an annular ring at the beginning (lower) end of the channel. The capillary channel is $600 \mu \mathrm{m}$ wide and about $15 \mathrm{~cm}$ long. The channel and the target chamber are filled with $\mathrm{Ne} / \mathrm{Xe}=99.9 / 0.1$ at one atmospheric pressure. Eight locations (A-H) are marked along the channel for reference of the locations of the ionization wave front. The full computational domain is a square of side length $12 \mathrm{~cm}$ whose boundaries are grounded except for the top (target) surface.

filling the tube and target chamber at $244 \mathrm{~ns}$ after initiation, and at locations along the channel as the IW front arrives. The electron density is approximately $5 \times 10^{13}-1 \times 10^{14} \mathrm{~cm}^{-3}$ throughout the length of the capillary. The electron density is 


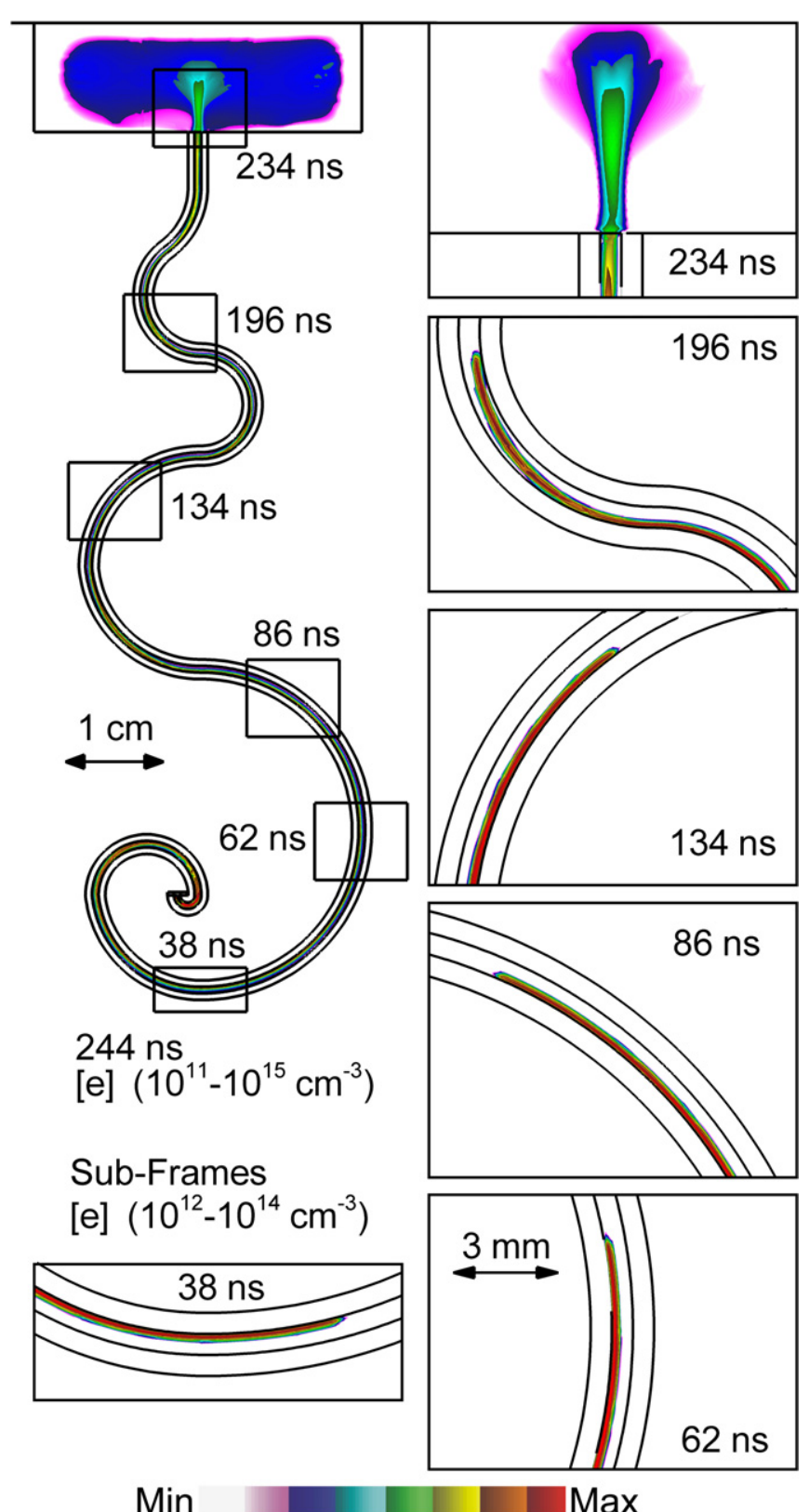

Figure 2. Electron density contours for the positive ionization wave $(50 \mathrm{kV})$ and streamer discharge before the impingement onto the surface. The electron density is shown filling the tube and target chamber at $244 \mathrm{~ns}$ after initiation, and at locations along the channel as the IW front arrives. The contours are on a log scale over the indicated ranges.

higher $\left((1-2) \times 10^{15} \mathrm{~cm}^{-3}\right)$ in the immediate vicinity of the powered electrode, and $1 \times 10^{13} \mathrm{~cm}^{-3}$ in the plume beyond the end of the capillary tube. Simulations were performed on channel lengths as high as $50 \mathrm{~cm}$, as demonstrated in experiments, and the IW front propagated with nearly the same characteristics as shown here [6]. As discussed below, the speed of the IW is $(1-3) \times 10^{7} \mathrm{~cm} \mathrm{~s}^{-1}$. As the head of the IW propagates through the channel, its properties (density, temperature and speed) oscillate about a mean value that depends on the local topology. However visually, the IW appears unchanged as it winds its way through the channel. In sections of the tube that are curved, the electron distribution

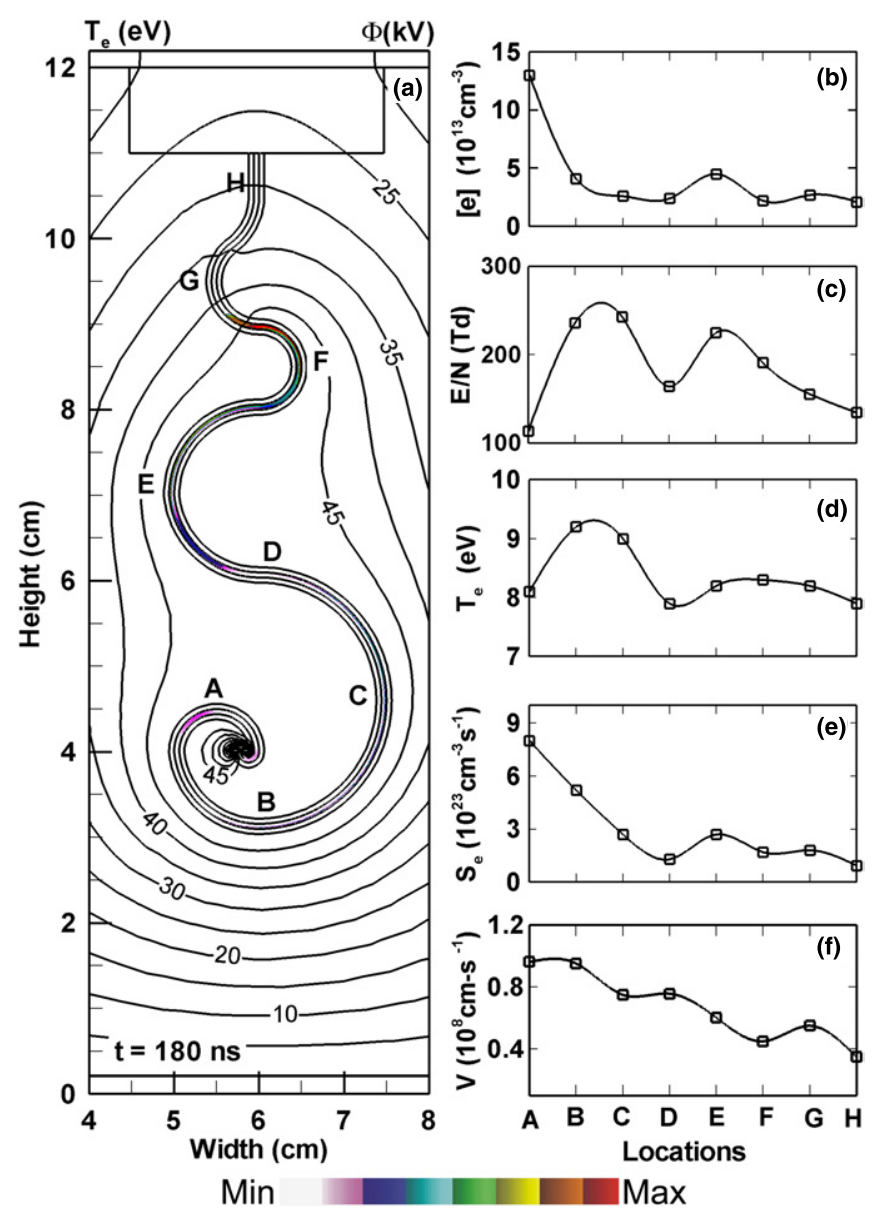

Figure 3. Properties of a positive IW generated by a $50 \mathrm{kV}$ voltage pulse. (a) Contours of electron temperature (flood) and electric potential (lines) at $t=180 \mathrm{~ns}$. $T_{\mathrm{e}}$ has a long extended tail of many centimeters following the ionization front inside the capillary. Quantities characterizing the ionization wave as it passes through different channel locations (points A-H) are shown on the right: $(b)$ electron density, $(c)$ reduced electric field, $(d)$ electron temperature, $(e)$ ionization source function and $(f)$ instantaneous speed.

of the IW hugs the wall [6], generally on the side of the wall with a smaller radius of curvature, and has a width of about $300 \mu \mathrm{m}$. In transition sections where the curvature changes, as at $196 \mathrm{~ns}$, the IW front flips from one side of the channel to the other. It is only in perfectly straight sections, as at the end of the tube at $234 \mathrm{~ns}$, that the IW front is symmetrically centered in the channel.

The electron temperature, $T_{\mathrm{e}}$, and electric potential, $\Phi$, for the positive IW at $t=180 \mathrm{~ns}$ are shown in figure 3(a). Similar to an unconfined streamer, the highest value of $T_{\mathrm{e}}$, about $8 \mathrm{eV}$, occurs at the ionization front due to the intense spacecharge-enhanced electric field. However, the confinement of the channel walls gives rise to a much slower variation of $T_{\mathrm{e}}$ following the ionization front. For example, it takes several centimeters of plasma column trailing the ionization front before $T_{\mathrm{e}}$ drops to $1 \mathrm{eV}$. For unconfined streamers, similar decreases in $T_{\mathrm{e}}$ typically occur within a distance of millimeters or shorter. Other quantities, such as ionization source function, also show similar elongated distributions. In unconfined streamers, charged particle losses in the direction perpendicular to propagation are due only to gas phase 


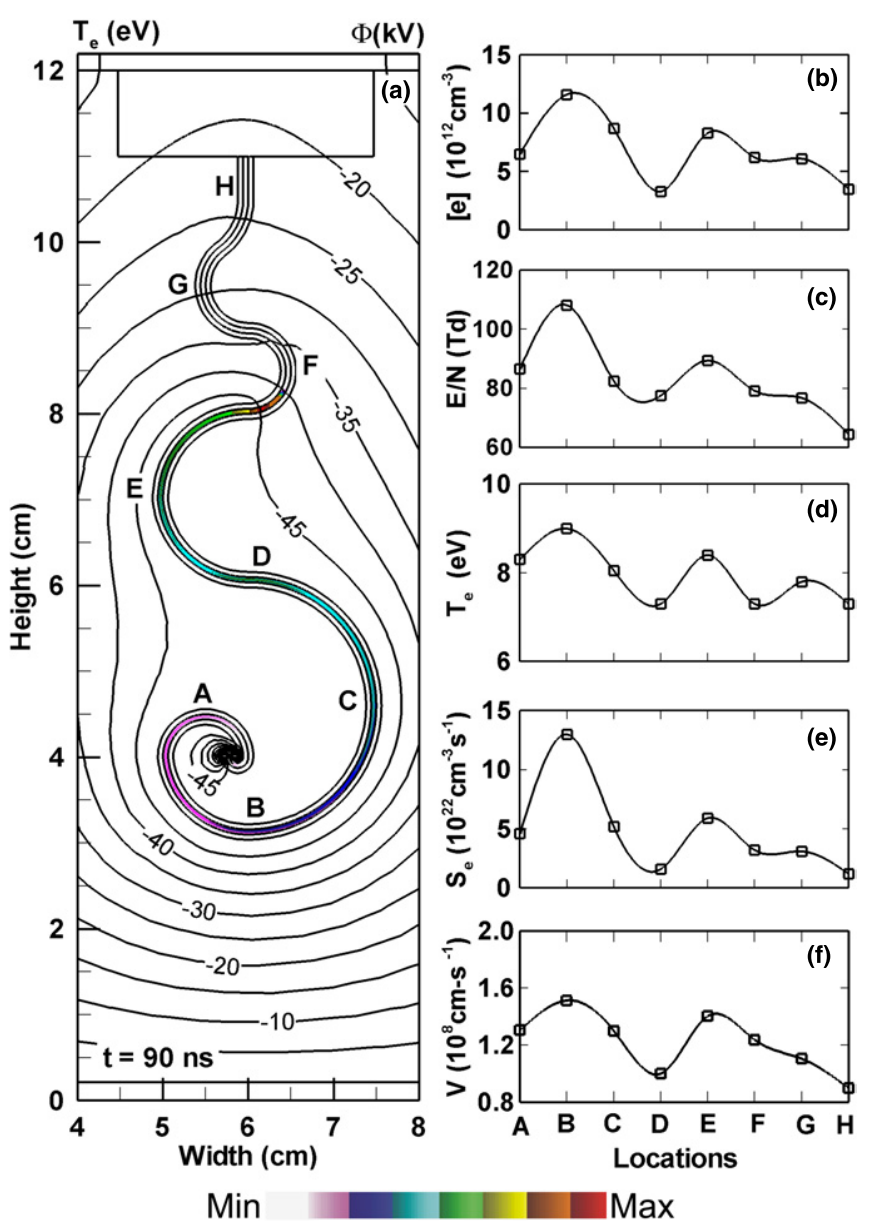

Figure 4. Properties of a negative IW generated by a $50 \mathrm{kV}$ voltage pulse. (a) Contours of electron temperature (flood) and electric potential (lines) at $t=90 \mathrm{~ns}$. Quantities characterizing the ionization wave as it passes through different channel locations (points A-H) are shown on the right: $(b)$ electron density, $(c)$ reduced electric field, $(d)$ electron temperature, $(e)$ ionization source function and $(f)$ instantaneous speed. The electron temperature has a longer tail in the capillary compared with the positive IW.

processes such as attachment or recombination. In capillary tubes having widths equal to the width of the unconfined streamer, there are additional and continuous losses of charge to the side walls. This increases the impedance of the plasma and hence increases the electric field behind the ionization front. Although the plasma is conductive and shorts out the potential along the path of the plasma, there is a finite voltage drop of about $500 \mathrm{~V}$ in the plasma channel shown in figure $3(a)$. The voltage drop across the ionization front itself is about $5 \mathrm{kV}$.

As the IW passes through the capillary channel, its properties are modulated by the curvature of the channel. To illustrate these dependences, plasma properties are noted at eight locations along the channel (marked by $\mathrm{A}-\mathrm{H}$ as in figures 1, 3 and 4) as the wave front, defined by the peak of the ionization source function, passes by. The electron density $n_{\mathrm{e}}$, normalized electric field $E / N$, electron temperature $T_{\mathrm{e}}$, electron impact ionization source function $S_{\mathrm{e}}$ and instantaneous wave speed $v_{\mathrm{w}}$ at points $\mathrm{A}-\mathrm{H}$ are plotted in figures $3(b)-(f)$. First, despite a general decreasing trend, the IW in the capillary largely maintains its strength and magnitude

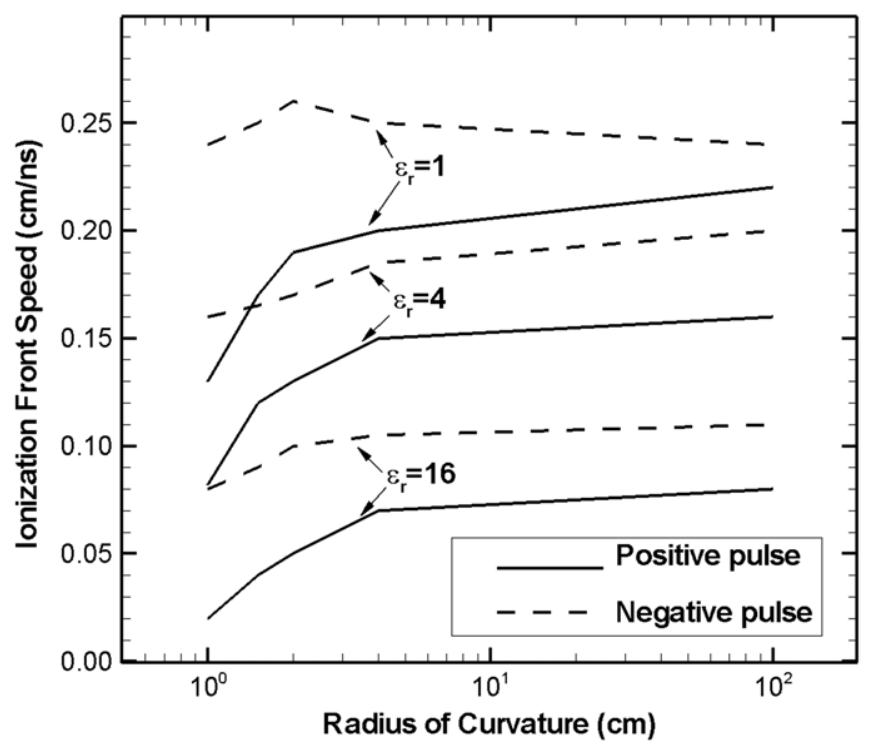

Figure 5. Speed of the ionization wave as a function of channel curvature and the dielectric constant of the wall. The channel width is $600 \mu \mathrm{m}$ with a length of $5 \mathrm{~cm}$ and bent with different radii of curvature. The wave speed decreases with increasing dielectric constant of the channel wall.

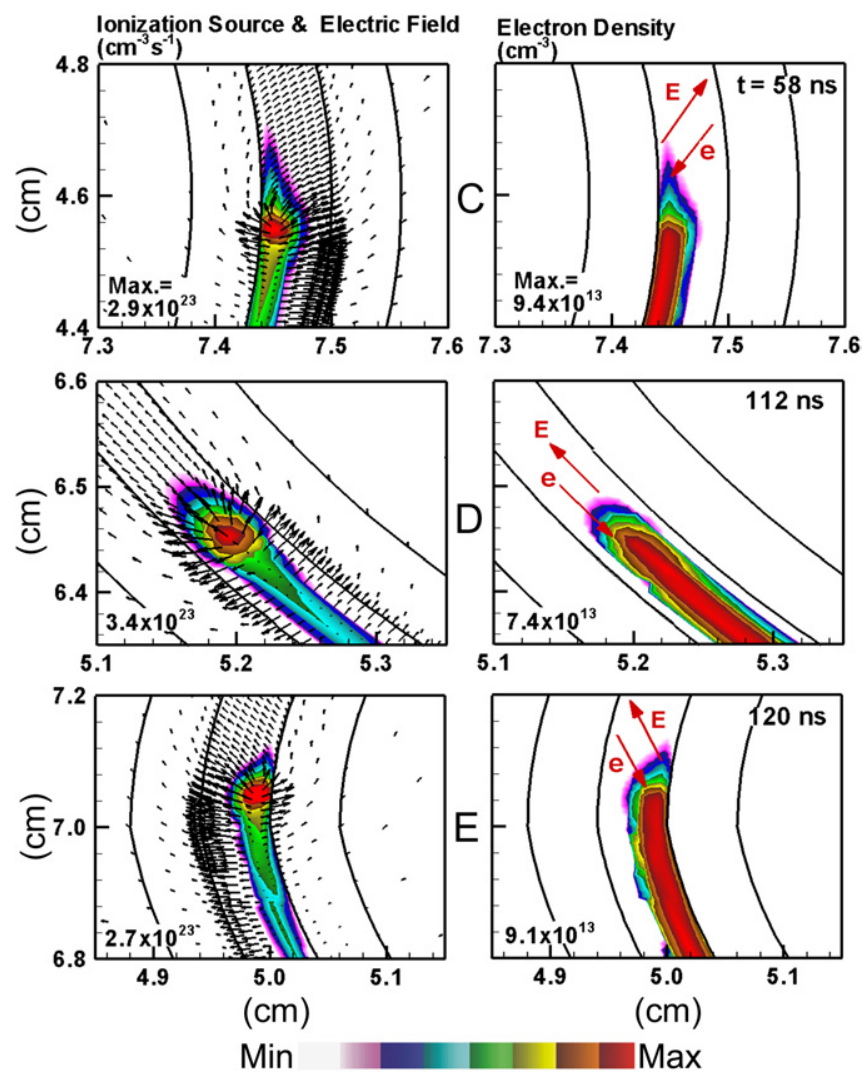

Figure 6. IW properties at different locations in the channel for a positive voltage $50 \mathrm{kV}$ as the ionization wave passes through points C, D, E (top to bottom). (a) Ionization source function and electric field; and $(b)$ electron density. The arrows on the electron density plots show the orientation of the instantaneous electric field in front of the IW and the drift direction of the photo-electrons. The contours for $n_{\mathrm{e}}$ and $S_{\mathrm{e}}$ are plotted on a log scale over 4 decades. 


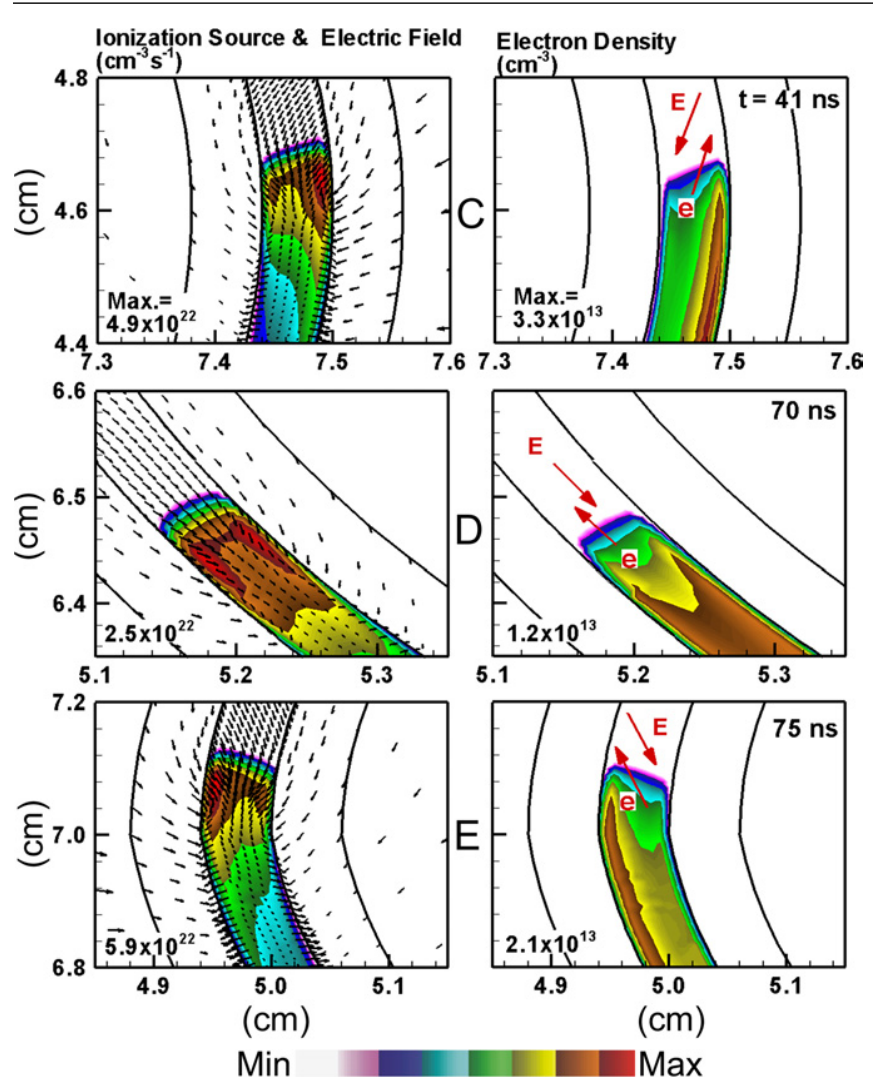

Figure 7. IW properties at different locations in the channel for a negative voltage $-50 \mathrm{kV}$ as the ionization wave passes through points C, D, E (top to bottom). (a) Ionization source function and electric field; and (b) electron density. The contours for $n_{\mathrm{e}}$ and $S_{\mathrm{e}}$ are plotted on a log scale over 3 decades.

within approximately a factor of $2-3$. This decrease is largely a result of the increasing voltage drop across the plasma along the length of the tube as the plasma column extends inside the channel. The reduced electric field $E / N$ at point $\mathrm{F}, \approx 180 \mathrm{Td}$, decreases by about $20 \%$ from its peak value at point $\mathrm{B}$ while the vertical distance from the voltage source increases by a factor of 5. The relatively small changes in $T_{\mathrm{e}}$ reflect these slowly varying values of $E / N$. This ability to maintain a nearly constant plasma source function for the length of the channel, in spite of the circuitous path, is critical for using capillary tubes to deliver excited states, radicals and ions to remote locations.

Second, none of the quantities varies monotonically along the length of the channel. As the IW propagates, the applied voltage (with some decrease due to plasma resistance) is expended across the instantaneous ionization front with electric field enhancement due to the space charge produced by the ionization front itself. With a curved circuitous channel, there are additional plasma surface interactions that add complexity to the propagation. From figures $3(b)-(f)$, and particularly from the behavior of $T_{\mathrm{e}}$ and $S_{\mathrm{e}}$, the propagation of the IW appears to have two stages. The initial development of the IW (before point D) is strongly influenced by the voltage source, showing rapid changes in $n_{\mathrm{e}}, T_{\mathrm{e}}$ and $S_{\mathrm{e}}$. This is likely a geometrical effect resulting from the rapid reduction in the electric field enhancement due to the finite extent and curvature of the electrode. Further extension of the IW beyond point D

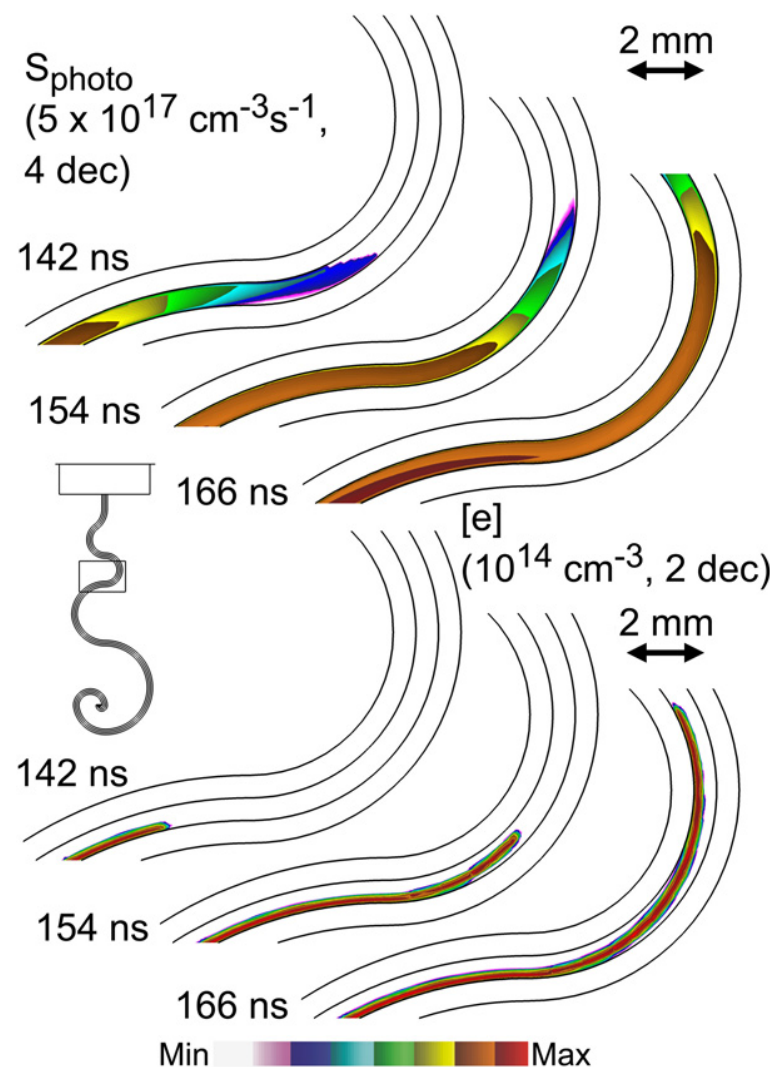

Figure 8. Photoionization source $S_{\mathrm{p}}$ and electron density $n_{\mathrm{e}}$ as the positive IW front flips from one side of the channel to the other near point F at $t=142,154$ and $166 \mathrm{~ns}$. Note the shadowing of $S_{\mathrm{p}}$ as the IW negotiates the curves. The contours are on a log scale over the indicated ranges.

becomes less dependent on the details of the topography of the channel and location of the voltage source. Past this point, the IW enters into a quasi-steady state with gradual variations of its properties. These development stages are qualitatively consistent with experiments in [6].

Third, the IW speed is in the range (3-10) $\times 10^{7} \mathrm{~cm} \mathrm{~s}^{-1}$, in agreement with experiments by Robert et al [6]. Despite a general decreasing trend in speed along the channel, there are local variations in speed dependent on the local radius of curvature of the channel. Smaller radii of curvature tend to have larger $E / N, T_{\mathrm{e}}$ and $S_{\mathrm{e}}$, but lower wave speed. The larger $E / N$ and $T_{\mathrm{e}}$ and $S_{\mathrm{e}}$ are likely due to electric field enhancement due to the curvature of the channel in the presence of a gradient in permittivity across the channel-plasma boundary. The slowing of the wave speed $v_{\mathrm{w}}$ has multiple causes. One is the increased capacitance of the curved channel compared with a straight channel. As discussed below, a longer dwell time of the plasma is required to charge the larger capacitance of the wall. The dependence of $v_{\mathrm{w}}$ may also be explained by the influence of photoionization which produces seed electrons in front of the IW. For positive IWs, it is generally known that increasing the rates of photoionization ahead of the IW increases the speed of the IW. In that regard, the view-angle for photoionizing radiation ahead of the IW is larger on relatively straight channel sections compared with highly curved channels, and hence one might expect higher speeds along channel sections with larger radii of curvature. 


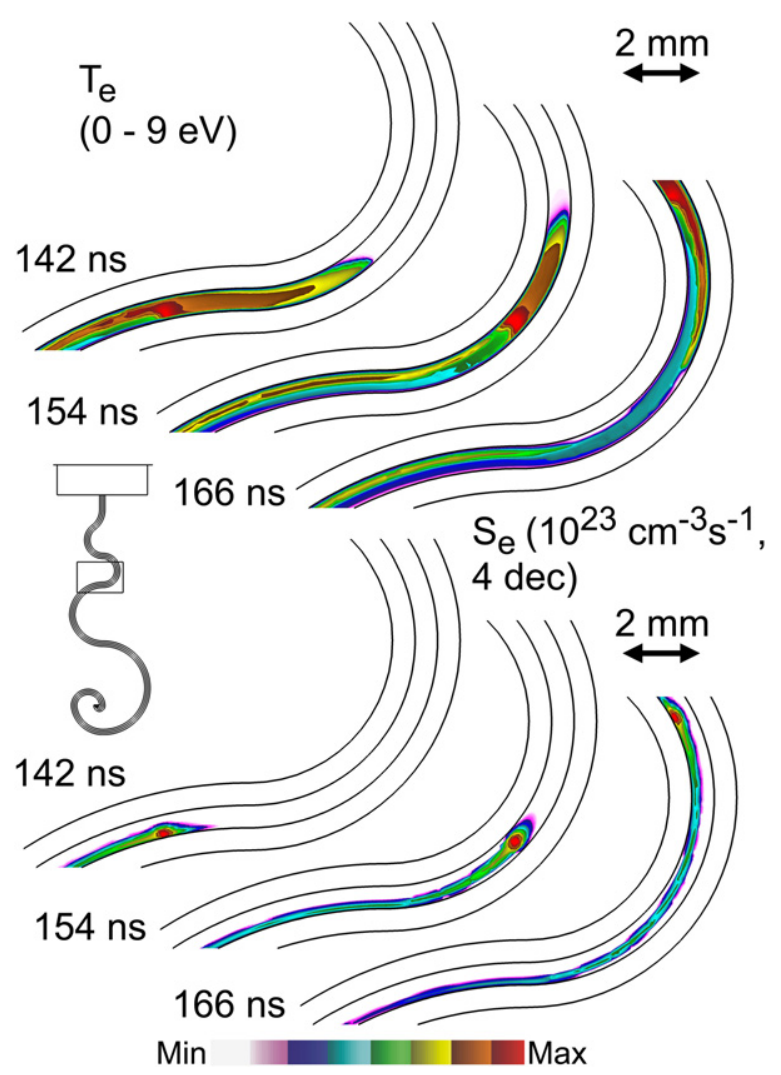

Figure 9. Electron temperature $T_{\mathrm{e}}$ and electron impact ionization source $S_{\mathrm{e}}$ as the positive IW front flips from one side of the channel to the other near point $\mathrm{F}$ at $t=142,154$ and $166 \mathrm{~ns}$. A region of elevated $T_{\mathrm{e}}$ extends ahead of the IW consisting of photoionization generated electrons. $S_{\mathrm{e}}$ contours are on a log scale over the indicated range.

$T_{\mathrm{e}}$ is shown in figure 4, along with $E / N, n_{\mathrm{e}}, T_{\mathrm{e}}, S_{\mathrm{e}}$ and $v_{\mathrm{w}}$ at stations along the channel for a negative polarity pulse $(-50 \mathrm{kV})$. The peak value of $T_{\mathrm{e}}$ is near $8 \mathrm{eV}$ and its distribution along the channel is similar to that of the positive case. Other plasma properties, $n_{\mathrm{e}}, E / N, S_{\mathrm{e}}$ and $v_{\mathrm{w}}$, as in the positive case, maintain their values throughout the channel (after the startup transient), with a gradual downward trend. However, there are some quantitative differences from the positive pulse.

First, the average $n_{\mathrm{e}}$ at the ionization front for negative polarity is nearly one order of magnitude lower than that for the positive case, as is the ionization source $S_{\mathrm{e}}$. The average value of $E / N$ in the IW is also reduced by about half compared with the positive case. The reduced electron density results in a more resistive plasma channel for the negative polarity. The potential drop within the plasma channel is about $3-4 \mathrm{kV}$, as compared with $0.5 \mathrm{kV}$ for a similar channel length in the positive case. This also leads to an even longer tail of $T_{\mathrm{e}}$ than in the positive pulse case, approaching $10 \mathrm{~cm}$ and extending almost to the beginning of the channel, as shown in figure 4(a). These characteristics are similar to the polarity effects observed in unconfined streamers, where negative streamers tend to have a more extended ionization channel behind the streamer head than the positive ones $[18,23]$.

Second, the propagation of the negative IW is basically determined by the local $E / N$, with much less contribution from photoionization compared with the positive case. So unlike the positive IW, the variations of $n_{\mathrm{e}}, T_{\mathrm{e}}, S_{\mathrm{e}}$ and $v_{\mathrm{w}}$ for

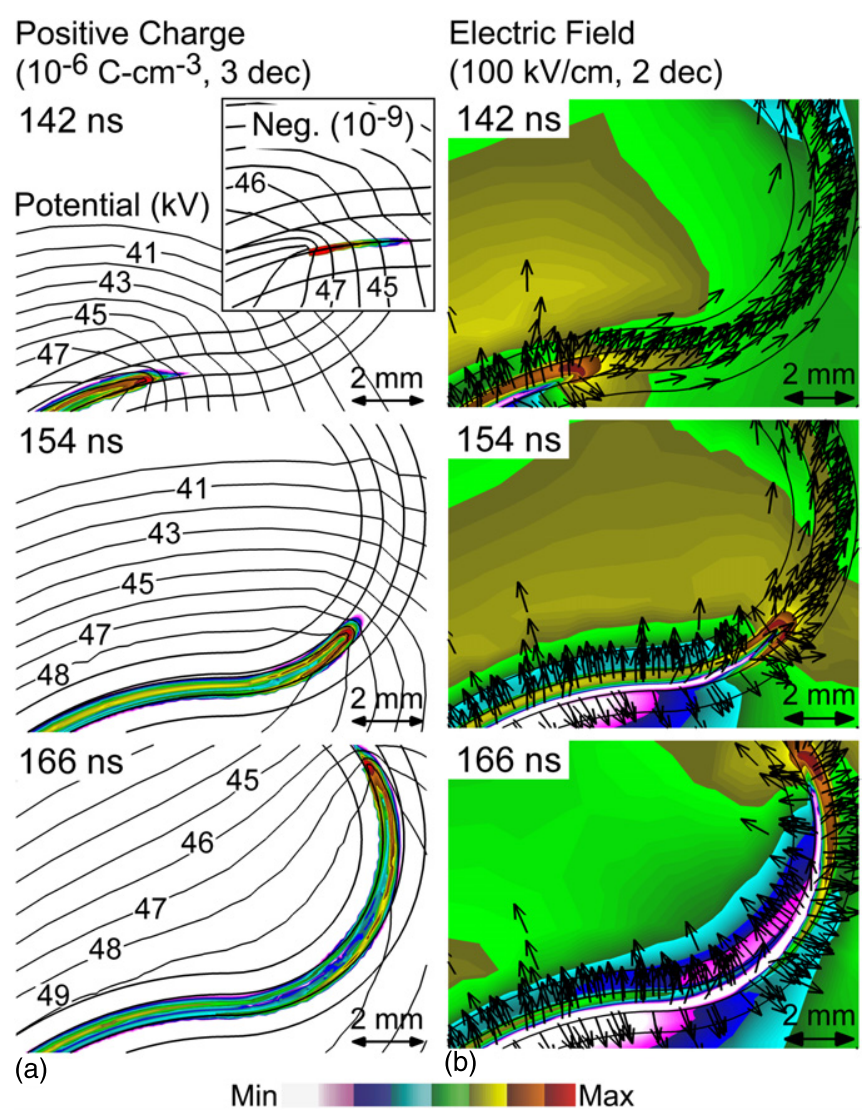

Figure 10. Positive charge density with electric potential contours and the electric field (and vectors) as the positive IW front passes near point $\mathrm{F}$ at $t=142,154$ and $166 \mathrm{~ns}$. The electric field vectors show only the direction but not the magnitude of the field. The positive charge and electric fields are plotted on a log scale over the indicated number of decades. The inset shows negative charge ahead of the streamer.

negative IW, as shown in figures $4(b)-(f)$, are almost directly correlated with the variations in the local $E / N$. That is, the shadowing effect of photoionization by the curvature of the channel is less important to the propagation speed.

Third, in spite of the decreases in $n_{\mathrm{e}}$ and $E / N$, the average propagation speed through the channel for negative IWs increases by approximately a factor of 2 , to $1.2 \times 10^{8} \mathrm{~cm} \mathrm{~s}^{-1}$, compared with the positive case. This is in contrast to the plasma jets or streamers in open air where the IWs with negative polarity typically propagate slower than the positive ones $[15,17,23]$. This relative difference in IW speeds at different polarities, in unconfined space and capillary tubes, may be explained to a certain extent by the electron drift, which is the major charge transport mechanism during the short time scale of the IW. For negative polarity, the electrons drift and diffuse in the direction of wave propagation which reduces the peak value of electron density at the ionization front, reduces the charge density and hence produces a lower value of $E / N$. Conversely, for positive polarity, the electrons drift against the direction of IW propagation, thereby compressing the ionization front, producing a higher charge density and larger $E / N$. The larger $E / N$ produces a higher rate of ionization and higher peak electron density. Thus, in unconfined space, the negative streamers typically propagate slower than the positive ones. 


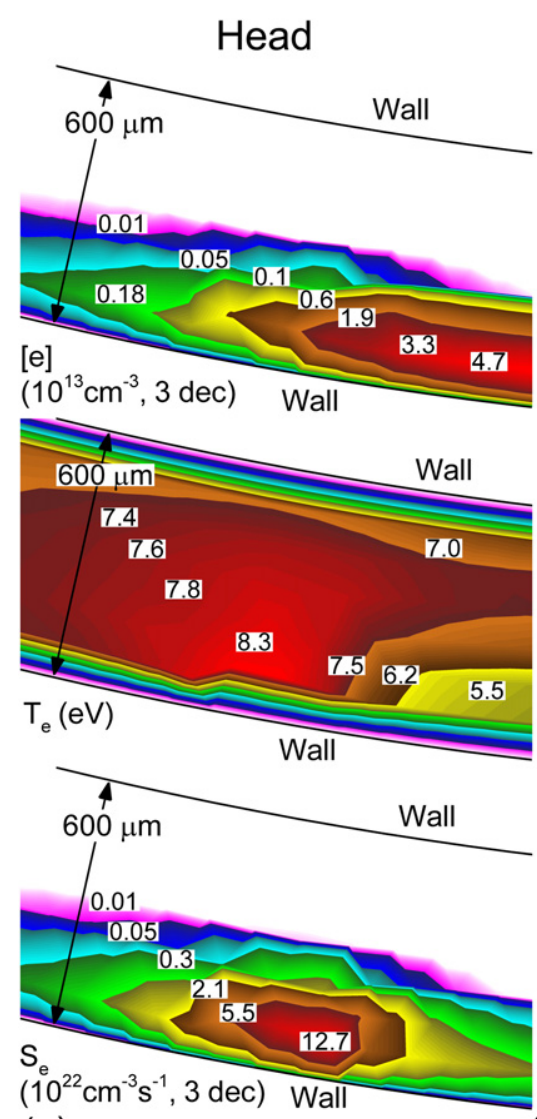

(a)
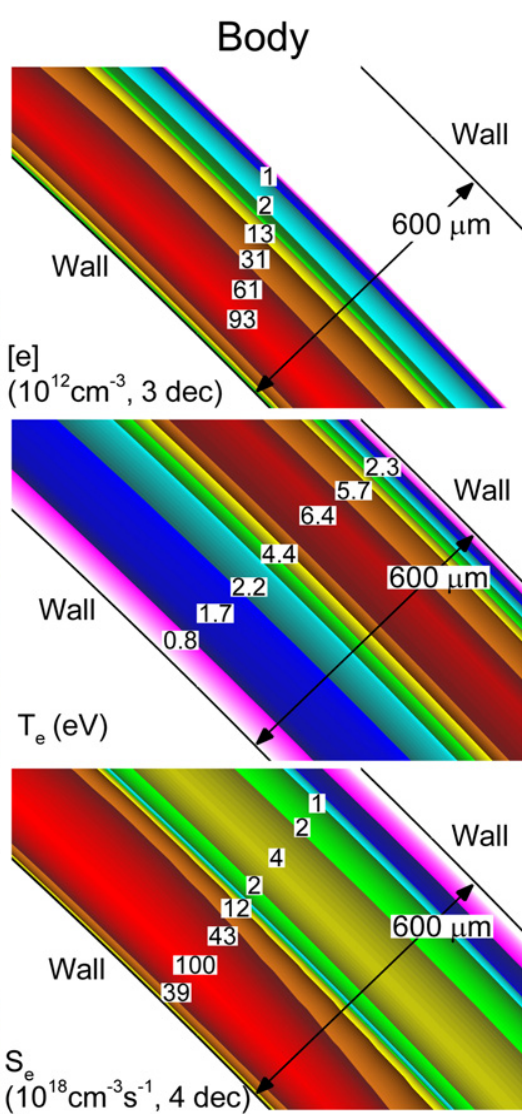

(b)

\section{Min $\quad$ Max}

Figure 11. Structure of the in-channel streamer at the head (left) and the body (right) approximately $1 \mathrm{~cm}$ behind the head. The contours for $n_{\mathrm{e}}$ and $S_{\mathrm{e}}$ are plotted on a log scale over the indicated number of decades. The contour labels are multipliers of the normalization value shown in each frame.

In capillary tubes, on the other hand, the presence of the confining dielectric walls provides a focusing effect to the IWs, particularly for the negative polarity IWs through the fast electron diffusion onto the walls. Despite the smaller $E / N$, in a confined space like a capillary tube, the electron drift in the negative polarity case produces sufficiently high seed electron densities in front of the IW so that there is less dependence on photoionization, a process which is sensitive to the curvature of the channel. The seeding of electrons in front of the IW by electron drift in the capillary tubes typically dominates that by photoionization, hence the speed in the negative polarity pulse is nearly twice as that in the positive case. This may also explain why there is less dependence of plasma properties on the curvature of the channel.

The speed of the IW is shown in figure 5 as a function of permittivity of the channel wall and radius of curvature of the channel. These results were obtained for a short $(5 \mathrm{~cm})$ length of tubing with a single bend of the specified curvature. In general, $v_{\mathrm{w}}$ decreases with increasing $\varepsilon / \varepsilon_{0}$ for both positive and negative pulses. These are trends observed for straight tubes [19] and are predicted analytically [11]. In order for the IW to propagate along the channel, the direction of the electric field must nominally be oriented parallel to the axis. This redirection of the orientation of the vacuum electric field to be aligned along the axis occurs in large part due to the charging of the walls of the channel, a process analogous to the breakdown of low-pressure glow discharges, such as fluorescent lamps [24], in dielectric tubes. The larger the dielectric constant and capacitance of the walls of the channel, the longer the dwell time of the plasma needs to be at a given location to charge that capacitance. Since the IW will not propagate along the axis unless there is a component of the electric field in that direction, pre-charging or at least simultaneous charging of the walls is required to reorient the electric field along the axis in order for propagation to continue along the circuitous path.

The speed of the IW is also a function of the radius of curvature of the channel, also shown in figure 5. Smaller radii of curvature produce lower propagation speeds for the IW, a consequence partly resulting from the larger capacitance of the curved surface. Positive IWs are more sensitive to the radius of curvature than negative IWs, an effect we attribute to the speed of positive IWs being more sensitive to the shadowing of ionizing radiation by the curvature of the tube.

Inside the channel, the peaks of $S_{\mathrm{e}}$ and $n_{\mathrm{e}}$ tend to propagate along the walls of the channel rather than filling the channel. They also tend to switch to the opposite side of the wall when the curvature of the channel changes. This surface-bound propagation pattern has also been observed in the experiments [6] and under select conditions in straight channels [20]. $S_{\mathrm{e}}$, electric field and $n_{\mathrm{e}}$ are shown in figure 6 as the positive IW front passes through points $\mathrm{C}, \mathrm{D}$ and $\mathrm{E}$, where the channel curvature changes from positive, to nearly zero, to negative. 


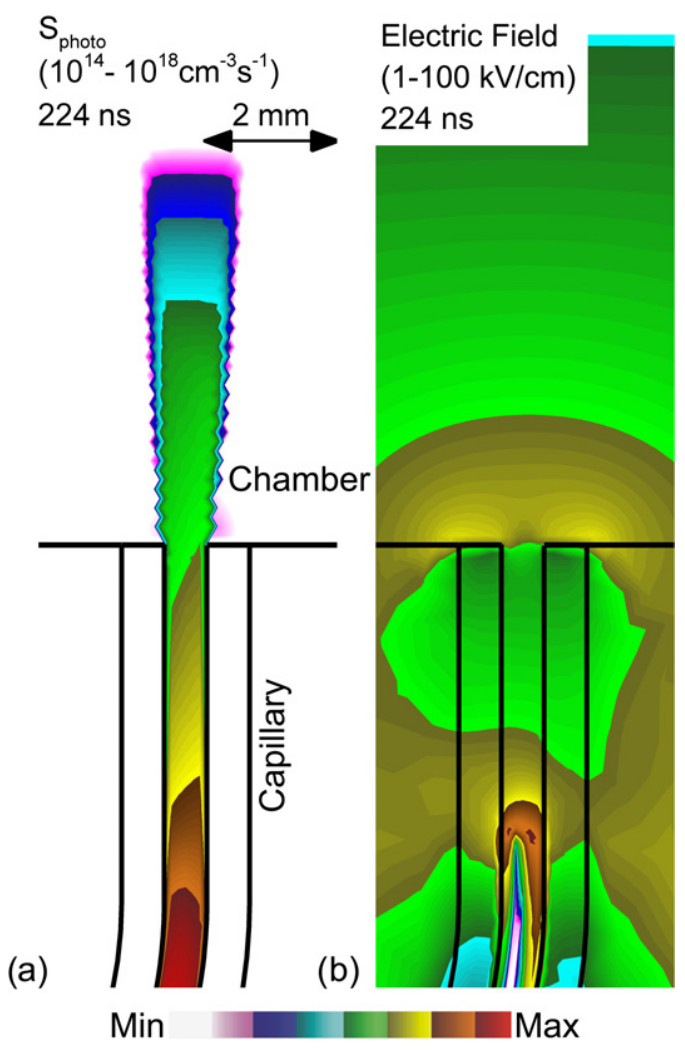

Figure 12. Seeding of the streamer in the chamber. $(a)$ Photoionization source and $(b)$ electric field at $224 \mathrm{~ns}$ as the positive IW approaches the mouth of the capillary tube. Note the curved front of the electric field in the IW and the enhancement of the electric field at the mouth of the tube. The photoionization source has an asymmetry due to view-angle that favors the right side. The contours are plotted on a log scale over the indicated range. The serrated edge in photoionization is an artifact of the plotting and not physical.

The peak locations of the ionization and electron density change from the inner (left) wall at the point $\mathrm{C}$, to the middle at $\mathrm{D}$, and finally to the outside (right) wall at $\mathrm{E}$. The reverse order from outside to inside holds true for the negative polarity, as shown in figure 7 . This change in the locations of the IW coincides with the change in the relative orientation between the electric field in front of the IW and the local capillary channel itself. The peak ionization and electron density occur at the wall where the local electric field points away from the wall and into the plasma, such as points $\mathrm{C}$ and $\mathrm{E}$. When the local electric field is aligned with the channel, such as at point $\mathrm{D}$, the ionization peaks move to the center of the channel. The same observations hold for the negative polarity as shown in figure 7 .

The dynamics of the flip of the IW front from one side of the channel to the other, while passing through point $\mathrm{F}$, are shown in figure 8 (photoionization source $S_{\mathrm{p}}$ and $n_{\mathrm{e}}$ ) and figure $9\left(T_{\mathrm{e}}\right.$ and $\left.S_{\mathrm{e}}\right)$. The IW front initially hugs the lower wall. Radiation from $\mathrm{Ne}_{2}^{*}$ produces ionization throughout the volume of the channel, since its absorption length significantly exceeds the channel width. When the IW front is below the bend in the channel, $S_{\mathrm{p}}$ is shadowed and so photoionization is dominantly along the lower wall and hence reinforces the front hugging the lower wall. $S_{\mathrm{p}}$ extends a few millimeters ahead of the IW head, producing a background electron density of $10^{9}-10^{10} \mathrm{~cm}^{-3}$. This density is too low to support sufficient charge density to produce an IW front. The background electrons are heated to $4-5 \mathrm{eV}$ by the vacuum fields up to a few millimeters ahead of the IW front contributing to ionization at low levels $\left(S_{\mathrm{e}}<10^{19} \mathrm{~cm}^{-3} \mathrm{~s}^{-1}\right)$. The region of highest ionization in the IW head, $S_{\mathrm{e}} \approx 10^{23} \mathrm{~cm}^{-3} \mathrm{~s}^{-1}$, has a length of $500-600 \mu \mathrm{m}$, with a tail that extends in excess of $1 \mathrm{~cm}$, progressively decreasing in magnitude to $10^{19} \mathrm{~cm}^{-3} \mathrm{~s}^{-1}$. As the curvature of the channel changes sign, photoionization illuminates the upper wall, which precedes the change in side of the channel of the head of the IW.

As mentioned above, charging of the wall of the channel changes the direction of the electric field from the vacuum configuration to approximately being aligned with the axis of the channel. Small deviations of the electric field from being aligned with the axis of the channel then accounts, in part, for the wall hugging IW. The charge density in the channel and the electric field are shown as the IW front rounds point $F$ in figure 10. The electric potentials are also shown as contour lines. The electric field vectors show only the direction, not the magnitude. The charge density is maximum at the center of the IW at $\rho=10^{-6} \mathrm{C} \mathrm{cm}^{-3} \mathrm{~s}^{-1} \approx q\left[6 \times 10^{13} \mathrm{~cm}^{-3}\right]$, producing an electric field of $100 \mathrm{kV} \mathrm{cm}^{-1}$ (or $400 \mathrm{Td}, 1 \mathrm{Td}=$ $10^{-17} \mathrm{~V} \mathrm{~cm}^{2}$ ). As a conductive plasma column is formed, positive charge is deposited on the channel walls whereas the plasma column is nearly quasi-neutral. A small amount of negative charge is deposited on the sidewalls ahead of the main IW body. The negative charging results from photoionizationproduced electrons ahead of the IW which freely diffuse to the walls. As the walls charge and the main IW body becomes conductive, the direction of the electric field vectors changes in two stages: the first is from their vacuum orientation to being approximately aligned with the axis of the channel; the second is from being aligned with the axis to being oriented perpendicular to the walls of the channel.

The structure of the IW differs markedly between its head and body. For example, $n_{\mathrm{e}}, T_{\mathrm{e}}$ and $S_{\mathrm{e}}$ are shown in figure 11 for the head and a position approximately $1 \mathrm{~cm}$ behind the head. The IW hugs the lower wall. In the head, $T_{\mathrm{e}}$ is maximum at $8 \mathrm{eV}$ about $400 \mu \mathrm{m}$ ahead of the maximum in $n_{\mathrm{e}}, 4.7 \times 10^{13} \mathrm{~cm}^{-3}$. The maximum in $S_{\mathrm{e}}, 1.3 \times 10^{23} \mathrm{~cm}^{-3} \mathrm{~s}^{-1}$, occurs between the peaks in $T_{\mathrm{e}}$ and $n_{\mathrm{e}}$, but they are all in alignment. In the body of the IW, the electron density in excess of $10^{9} \mathrm{~cm}^{-3}$ is confined within $300 \mu \mathrm{m}$ of the bottom wall. An electron density of a few $10^{9} \mathrm{~cm}^{-3}$ occupies the upper part of the channel, largely a consequence of photoionization. The high conductivity in the lower part of the channel shields out the electric field, resulting in a $T_{\mathrm{e}}$ of $1.5-3 \mathrm{eV}$. The maximum $T_{\mathrm{e}}$ of $6.5 \mathrm{eV}$ occurs in the low electron density portion of the channel. The end result is a weak electron source in the body of the IW that is about 1400 times smaller than in the head.

\section{Streamers in the target chamber}

When the IW exits the channel into the target chamber, it brings a large fraction of the applied potential to the junction of the channel and chamber. There are two effects that enhance 


\section{Ionization Source}

\section{Electron Density}

Dielectric-
Chamber
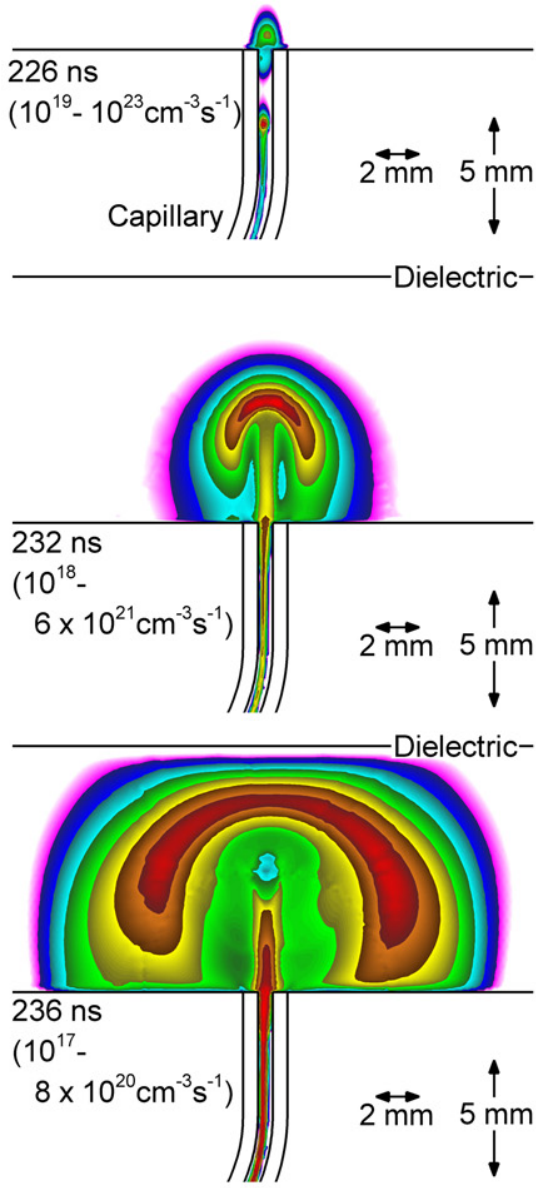

Dielectric-

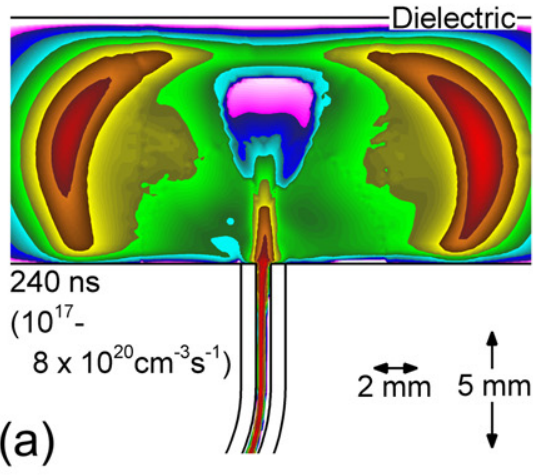

Min
Chamber

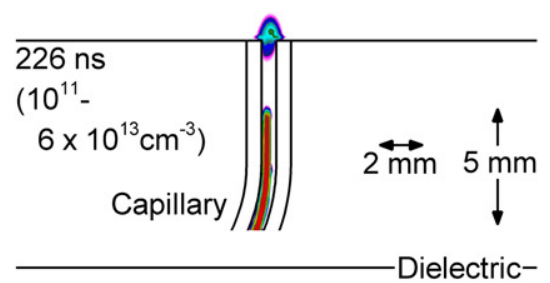

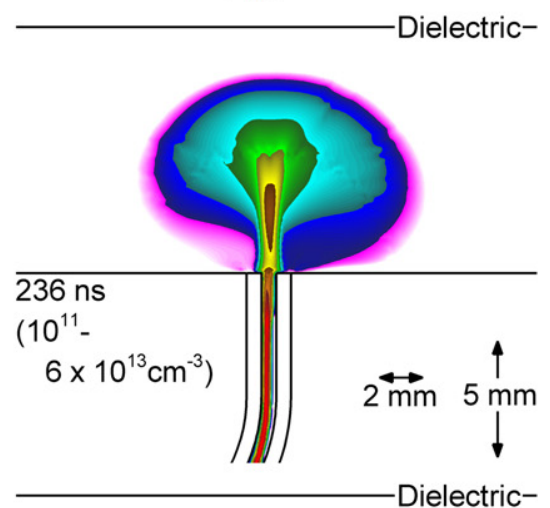

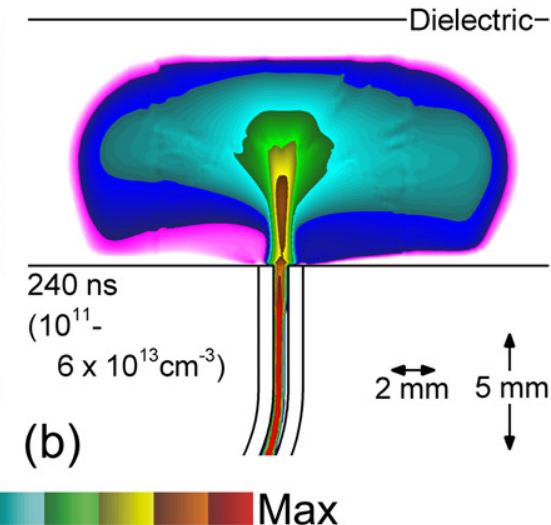

Figure 13. Development of streamer discharge in the target chamber induced by the approaching ionization wave of positive polarity from the capillary channel. (a) Ionization source function $S_{\mathrm{e}}$ and $(b)$ electron density $n_{\mathrm{e}}$ at times of 226, 232, 235 and 240 ns. The contours are plotted on a log scale over the indicated ranges.

the IW at this point. The first is that there is geometric electric field enhancement at the mouth of the channel resulting from the sharp edge and larger permittivity of the tube, as shown in figure 12. The approaching IW in the channel illuminates this region of enhanced electric field with VUV, producing photoionization, and launches a streamer inside the target chamber even before the ionization front itself exits the channel. Second, the reduction in losses from the ionization front, due to the lack of bounding walls, enables a rapid expansion of the ionization in the lateral direction. $S_{\mathrm{e}}$ and $n_{\mathrm{e}}$ are shown in the target chamber in figures 13 and 14 for positive and negative voltages. 


\section{Ionization Source} Dielectric-

Chamber
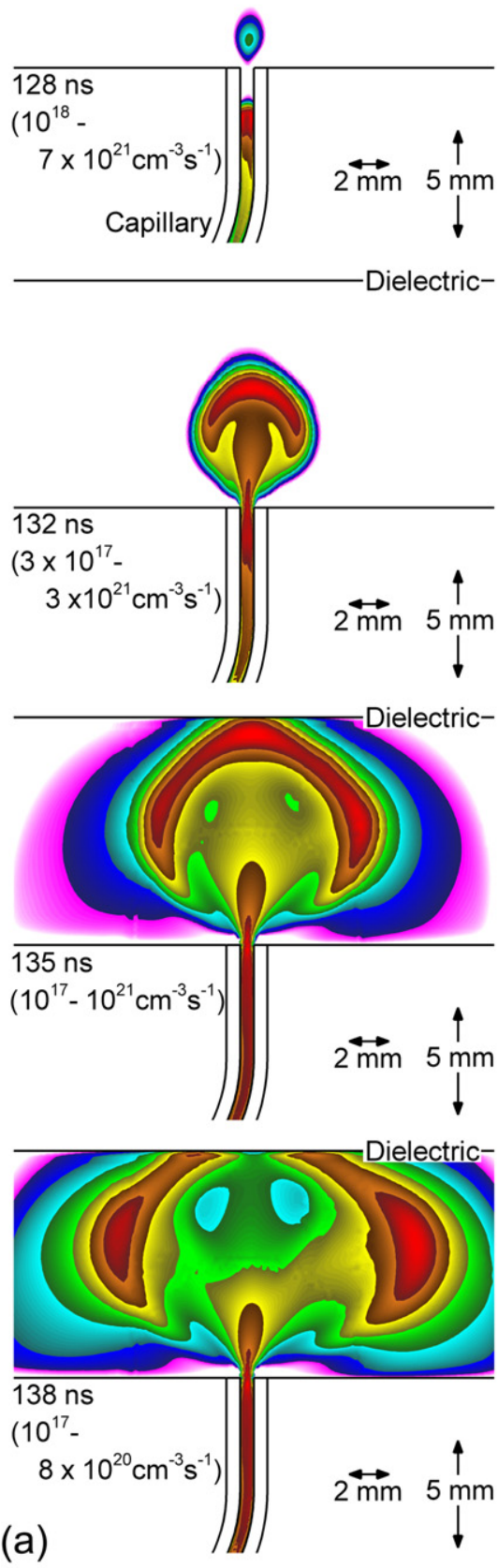

Min

\section{Electron Density}

Dielectric-

Chamber
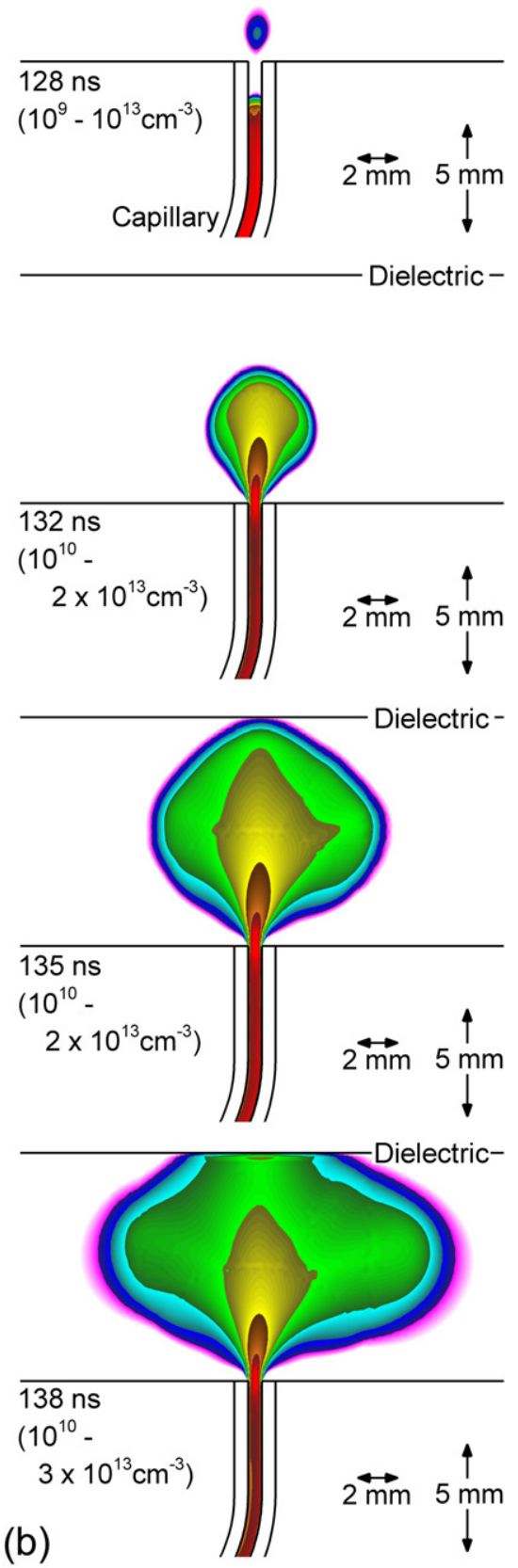

(b)

Max

Figure 14. Development of streamer discharge in the target chamber induced by the approaching ionization wave of negative polarity from the capillary channel. ( $a$ ) Ionization source function $S_{\mathrm{e}}$ and $(b)$ electron density $n_{\mathrm{e}}$ at times of $128,132,135$ and $138 \mathrm{~ns}$. The contours are plotted on a log scale over the indicated ranges.

For the positive polarity, a positive streamer is produced in the chamber at $t=226 \mathrm{~ns}$, seeded by photoionization from the approaching IW in the channel. Before the streamer impinges on the dielectric surface of the target, the region of ionization rate is fairly localized, as in a conventional streamer. The electron density in the chamber has an elongated, jet- like core well aligned with the channel exit with a circular outer distribution. The spreading of the plasma in a circular pattern agrees qualitatively with the experimental observations of helium plasma jets emerging into a helium chamber [25]. The more confined, jet-like plasma bullet results from the shroud of room air surrounding the gas jet emerging from 
the end of the tube, as discussed in [25]. The streamer speed in the chamber reaches about $1 \times 10^{8} \mathrm{~cm} \mathrm{~s}^{-1}$, which is about twice the speed of the IW at point $\mathrm{H}$ in the channel. However, the electron density in the chamber is less than that in the channel. The impingement of the streamer charges the target surface and splits the streamer into two which then propagates sideways with a similar intensity, similar to the spreading of a conventional DBD [26]. Note that there is slight asymmetry in both $S_{\mathrm{e}}$ and $n_{\mathrm{e}}$ which favors higher values (a few tens of percent) on the right side of the channel. This asymmetry results from asymmetric illumination of the mouth of the channel by the approaching IW. The line-of-sight of the approaching IW rounding the last curve favors the right side of the chamber.

The characteristics of the streamer in the target chamber for the negative polarity case are shown in figure 14 . A streamer discharge is also induced inside the target chamber by the approaching IW around $t=128 \mathrm{~ns}$ prior to the arrival of the IW from the channel. This is again due to the seeding of electrons by photoionization in the higher $E / N$ (with lower losses) at the mouth of the channel. The subsequent development of the streamer is qualitatively similar to that of the positive polarity case with a propagation speed of about $2 \times 10^{8} \mathrm{~cm} \mathrm{~s}^{-1}$, again nearly twice the speed of its IW precursor in the channel. The primary difference between the positive and negative cases is that the electron density in the latter case is more diffusive - the high electron density region is significantly wider than the channel. The diffusive feature of the negative streamer again results from the electron drift which is away from the ionization peak.

\section{Concluding remarks}

A numerical study of ionization waves propagating through a circuitous capillary channel and impinging upon a target, in the context of remote delivery of plasma species for biomedical applications, has been conducted. The capillary channel is about $15 \mathrm{~cm}$ long, $600 \mu \mathrm{m}$ wide (aspect ratio 250) consisting of sections of different radii of curvature. The channel is connected to a target chamber and both are filled with a gas mixture $\mathrm{Ne} / \mathrm{Xe}=99.9 / 0.1$ at $1 \mathrm{~atm}$. The ionization waves, initiated at the beginning of the channel by high-voltage $( \pm 50 \mathrm{kV})$ pulses, are able to propagate through the entire channel length while maintaining their magnitude and speed within factors of 2-3, and upon exiting, trigger streamer discharges in the target chamber which then impinge upon the target surface. Unlike the plasma bullets in open configurations, the IW fronts in the capillary channel are followed by an extended tail of high electron temperature and ionization up to several centimeters long. The average propagation speed in the channel is about $5 \times 10^{7} \mathrm{~cm} \mathrm{~s}^{-1}$ for positive ionization waves and $1 \times 10^{8} \mathrm{~cm} \mathrm{~s}^{-1}$ for negative IWs. The peaks of the ionization source function and electron density tend to propagate along one of the walls if there is any asymmetry rather than filling the channel. The IW also tends to switch to the opposite side of the wall when the channel curvature changes. This surface-bound propagation mode is found to coincide with the change in the relative orientation between the electric field in front of the wave front and the local capillary channel itself. In both positive and negative cases, the streamer in the target chamber is initiated prior to the arrival of the IW from the channel. The streamers then propagate at twice the speed of their IW precursors in the channel. The positive streamer has a more focused and jet-like core profile while the negative streamer is more diffusive. The impingement of the streamer charges the target surface and splits the streamer into two which then propagates sideways with a similar intensity. In addition to the properties discussed above, both the mode and speed of the ionization wave propagation can be modified by simply putting grounded objects near the capillary channel. The properties of the IWs produced with different voltages are found to be qualitatively similar to those presented here; however, the speed is lower for lower voltages.

\section{Acknowledgments}

This work was supported by the Department of Energy Office of Fusion Energy Sciences. We sincerely thank Drs Eric Robert and Jean-Michael Pouvesle for discussions on the topic of this paper and access to their experimental data.

\section{References}

[1] Laroussi M 2002 Nonthermal decontamination of biological media by atmospheric-pressure plasmas: review, analysis, and prospects IEEE Trans. Plasma Sci. 30 1409-15

[2] Stoffels E, Sakiyama Y and Graves D 2008 Cold atmospheric plasma: charged species and their interactions with cells and tissues IEEE Trans. Plasma Sci. 36 1441-57

[3] Fridman G, Friedman G, Gutsol A, Shekhter A B, Vasilets V N and Fridman A 2008 Applied plasma medicine Plasma Process. Polym. 5 503-33

[4] Kong M G, Kroesen G, Morfill G, Nosenko T, Shimizu T, van Dijk J and Zimmermann J L 2009 Plasma medicine: an introductory review New J. Phys. 11115012

[5] Kim J Y, Kim S, Wei Y and Li J 2010 A flexible cold microplasma jet using biocompatible dielectric tubes for cancer therapy Appl. Phys. Lett. 96203701

[6] Robert E, Barbosa E, Dozias S, Vandamme M, Cachoncinlle C, Viladrosa R and Pouvesle J M 2009 Experimental study of a compact nanosecond plasma gun Plasma Process. Polym. 6 795-802

[7] Hoffmann M, Bruch H, Kujath P and Limmer S 2010 Cold-plasma coagulation in the treatment of malignant pleural mesothelioma: results of a combined approach Interact. Cardiovasc. Thorac. Surg. 10 502-5

[8] Teschke M, Kedzierski J, Finantu-Dinu E, Korzec D and Engemann J 2005 High-speed photographs of a dielectric barrier atmospheric pressure plasma jet IEEE Trans. Plasma Sci. 33 310-1

[9] Mericam-Bourdet N, Laroussi M, Begum A and Karakas E 2009 Experimental investigations of plasma bullets J. Phys. D: Appl. Phys. 42055207

[10] Walsh J L, Iza F, Janson N B, Law V J and Kong M G 2010 Three distinct modes in a cold atmospheric pressure plasma jet J. Phys. D: Appl. Phys. 43075201

[11] Lagarkov A N and Rutkevich I M 1994 Ionization Waves in Electrical Breakdown of Gases (New York: Springer)

[12] Asinovsky E I, Lagarkov A N, Markovets V V and Rutkevich I M 1994 On the similarity of electric breakdown waves propagating in shielded discharge tubes Plasma Sources Sci. Technol. 3 556-63 
[13] Suzuki T 1977 Propagation of ionizing waves in glow discharge J. Appl. Phys. 48 5001-7

[14] Vasilyak L M, Kostyuchenko S V, Kudryavtsev N N and Filyugin I V 1994 Fast ionisation waves under electrical breakdown conditions Phys.-Usp. 37 247-69

[15] Luque A, Ratushnaya V and Ebert U 2008 Positive and negative streamers in ambient air: modeling evolution and velocities J. Phys. D: Appl. Phys. 41234005

[16] Naidis G V 2010 Modeling of streamer propagation in atmospheric-pressure helium plasma jets J. Phys. D: Appl. Phys. 43402001

[17] Naidis G V 2011 Simulation of streamers propagating along helium jets in ambient air: polarity-induced effects Appl. Phys. Lett. 98141501

[18] Georghiou G E, Papadakis A P, Morrow R and Metaxas A C 2005 Numerical modeling of atmospheric pressure gas discharges leading to plasma production J. Phys. D: Appl. Phys. 38 R303

[19] Jánský J, Tholin F, Bonaventura Z and Bourdon A 2010 Simulation of the discharge propagation in a capillary tube in air at atmospheric pressure J. Phys. D: Appl. Phys. 43395201

[20] Jánský J, Le Delliou P, Tholin F, Tardiveau P, Bourdon A and Pasquiers S 2011 Experimental and numerical study of the propagation of a discharge in a capillary tube in air at atmospheric pressure J. Phys. D: Appl. Phys. 44335201

[21] Xiong Z and Kushner M J 2010 Surface corona-bar discharges for production of pre-ionizing UV light for pulsed high pressure plasmas J. Phys. D: Appl. Phys. 43505204

[22] Takashima K, Adamovich I V, Xiong Z, Kushner M J, Czarnetzki U, Luggenhöscher D and Starikovskaia S 2011 Experimental and modeling analysis of fast ionization wave discharge propagation in a rectangular geometry Phys. Plasmas 18083505

[23] Jian C, Chen M T and Gundersen M A 2009 Polarity-induced asymmetric effects of nanosecond pulsed plasma jets J. Phys. D: Appl. Phys. 42232002

[24] Brok W J M, van Dijk J, Bowden M D, van der Mullen J J A M and Kroesen G M W 2003 A model study of propagation of the first ionization wave during breakdown in a straight tube containing argon J. Phys. D: Appl. Phys. 36 1967-79

[25] Zhu W-C, Li Q, Zhu X-M and Pu Y-K 2009 Characteristics of atmospheric pressure plasma jets emerging into ambient air and helium J. Phys. D: Appl. Phys. 42202002

[26] Xiong Z and Kushner M J 2011 Ionization wave splitting at the T-junction of a dielectric channel IEEE Trans. Plasma Sci. 392320 